\title{
X-RAY STUDIES OF REACTIONS IN
} ORGANIC SOLIDS

\author{
J. Z. Gougoutas
}

\author{
12 Oxford Street, Department of Chemistry, Harvard University, \\ Cambridge, Massachusetts 02138. USA
}

\begin{abstract}
Correlations of the chemical structures of products from organic solid state reactions with the molecular packing in the reactant crystal structures define the extent of lattice restraints on molecular motion before and during reaction. The molecular behaviour after the chemical transition state can be inferred from comparisons of the molecular orientations in the initial and final phases. The formation of preferentially oriented product phases suggests that highly specific geometrical mechanisms are operative but additional criteria are needed to establish a direct correspondence between specific reactant and product molecules. Observable intermediate mesomorphic phases are particularly useful in defining the directions in which crystallographic order is not preserved.

Several studies of hydrolysis, isomerization and polymerization reactions suggest surprisingly large molecular migrations and conformational changes may occur without randomizing the total distribution of product molecules. Competing and consecutive transformations often give rise to several simultaneously present and mutually oriented product phases and, in some cases, large molecular moieties of the initial structure do not appear in any of the final ordered phases. Novel twinning frequently is observed when degenerate transformation mechanisms give rise to several preferred orientations of product structures which cannot reorganize to form a single phase of long-range order. While the short-range molecular arrangement in the oriented product phase usually is identical with that produced during conventional crystallizations, exceptions may be found in the formation of chemically unstable molecular structures or metastable polymorphs of the product phase.
\end{abstract}

\section{INTRODUCTION}

In most reported examples of solid state reactions in molecular crystals, the products are structurally incompatible with the reactant lattice and eventually appear in a separate phase. The product phase usually is amorphous or polycrystalline and therefore has not retained the threedimensional periodicity of the reactant structure. The chemical reaction, nevertheless, may be quite specific. A wide variation in product states was observed in the extensive studies of solid state photochemical dimerization ${ }^{1}$ and no strong correlations are evident between the chemical specificity of the reaction and the overall crystallographic order of the final solid phase. 


\section{J. Z. GOUGOUTAS}

In these cases, it appears that the initial lattice may restrict selectively the modes of molecular motion before, but not after, the chemical transition state.

Topotactic reactions are notable exceptions $\dagger$. The product molecules assume preferred orientations relative to crystallographic directions of the reactant structure and it seems not unreasonable to suppose that the molecular motions at all stages of topotactic transformations are subject to the restraining effects of the continuously ordered reactant-product matrix. Even in these remarkable transformations, the product molecules virtually always crystallize during the reaction as a separate phase of characteristic cell dimensions and symmetry. The structure of the product phase can be determined and compared with the original phase according to the experimentally observed topotactic angular relationship. Although the topotaxy strictly defines only a reticular correspondence between the initial and final crystal structures, it is often meaningful to consider the phase transformation on a molecular level with the aim of establishing the geometrical mechanism by which the reactant molecules are displaced to their final lattice positions as product molecules of the chemical reaction.

The examples considered below illustrate the wide range of molecular motions which can occur without randomizing the final distribution of product molecules. In the extreme, a direct molecular correspondence is indicated between the initial and final phases. Initially adjacent reactant molecules are transformed to adjacent product molecules which 'crystallize' without further redistribution. The topotaxy reflects a chemical change and reorientation of only a small fraction of the total molecular bulk.

At the other extreme, an initially homogeneous reactant may undergo several different, competing and consecutive chemical reactions which result in many different, simultaneously present and mutually oriented crystal structures. Very extensive solid state redistribution occurs and, in some cases, large molecular moieties of the reactant structure do not appear in any of the final ordered phases. Product molecules which are adjacent or near neighbours in the final phase were relatively distant neighbours in the initial reactant structure. In view of the large displacements, it is not possible to identify particular product molecules with specific reactant molecules and therefore no information can be obtained on the intervening motions of the chemical reaction. The topotaxy clearly is not related simply to the chemical reaction but rather arises from a subsequent oriented growth of initially disoriented product molecules on one or several of the ordered phases.

\section{TOPOTAXY IN MOLECULAR CRYSTALS}

\section{Solid state polymerization}

The current scientific interest in topotactic processes in molecular crystals in no small way stems from their potential practical importance to the production of synthetic polymer fibres without recourse to mechanical orientation processes. The relatively brief history and development of

$\uparrow$ For a review of topotactic processes in inorganic solid state reactions, see reference 2 . 
topotactic polymerizations and polycondensations ${ }^{3}$ includes most reported cases of topotaxy in the organic solid state. Only some of the general crystallographic aspects of the transformations will be considered here with reference to two examples which raise interesting questions concerning the geometrical reaction mechanism.

The long polymer chains usually grow in several preferred directions of the monomer single crystal and multiple twinning is very common. Although the product phase frequently is fibrous and only unidimensionally oriented, several examples of three-dimensionally oriented polymers have been reported. The geometrical relationship between the end phases is determined from x-ray diffraction measurements of partially polymerized samples. The importance of lattice imperfections and surface effects, more correctly associated with epitactic reactions, is well recognized. General considerations of the crystallographic mechanism of growth are focused on correlations of polymer identity period with intermolecular spacings in growth directions of the monomer phase ${ }^{4}$.

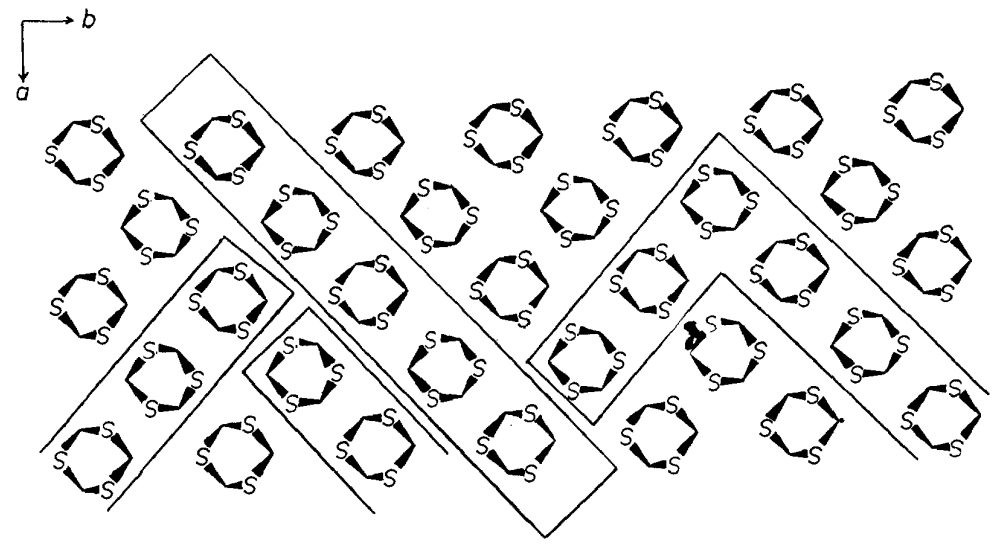

Figure 1. The molecular packing of trithiane in projection down [001]. The enclosed monomers indicate possible molecular combinations leading to the observed orientations of polymer chains

The origin of the ubiquitous twinning of product phase is evident in the study of three-dimensionally oriented polythiomethylene from single crystals of trithiane ${ }^{5}$. Two major and four minor preferred orientations of the helical polymer chains are indicated. The polymer crystal structure has a hexagonal cell with the polymer chain axis coincident with the sixfold axist. Polymerization of the orthorhombic monomer phase (space group $P m n 2_{1}$ ) presumably is propagated most favourably through the molecular contacts along the [110] diagonal since, after reaction, this direction defines the chain axis of the polymer structure (Figure 1). This direction is structurally unique in the polymer whereas, in the monomer, it is degenerate with [110]. Chain propagation accordingly occurs along both diagonals resulting in two orientations of polymer chains which together are incompatible with the known crystal structure of the polymer. Similarly, the four minor orientations

$\dagger$ A hexagonal cell was chosen for the assigned triclinic space group $P 1^{6}$. 


\section{J. Z. GOUGOUTAS}

of polymer chains are consistent with the fourfold multiplicity of a general line in the orthorhombic system. It is interesting to note that this type of twinning reflects the unique origin of the polymer crystal structure and apparently has not been observed in other preparations of the crystalline polymer.

The intermolecular distances in trithiane along the directions of preferred growth are less than necessary to accommodate the corresponding atoms of the polymer helix. It has been suggested that when this discrepancy becomes too severe from increasing strain, twinning occurs with growth in an alternative direction. The twinning may represent a geometrical relationship between individual crystallites comprised of straight polymer chains or, more interestingly, may result in a kink in the continuously growing chain.

Detailed considerations of the specific interactions which lead to reaction between trithiane molecules cannot be justified solely on the basis of the static monomer structure. Even small orientational changes of the highly symmetric structure during reaction may lead to several different modes of chain propagation. Such ambiguities were partially avoided in a study of the polymerization of vinyl stearate ${ }^{7}$. The reactive vinyl groups lie in planes separated by approximately $25 \AA$ (Figure 2 ) and it is reasonable to assume

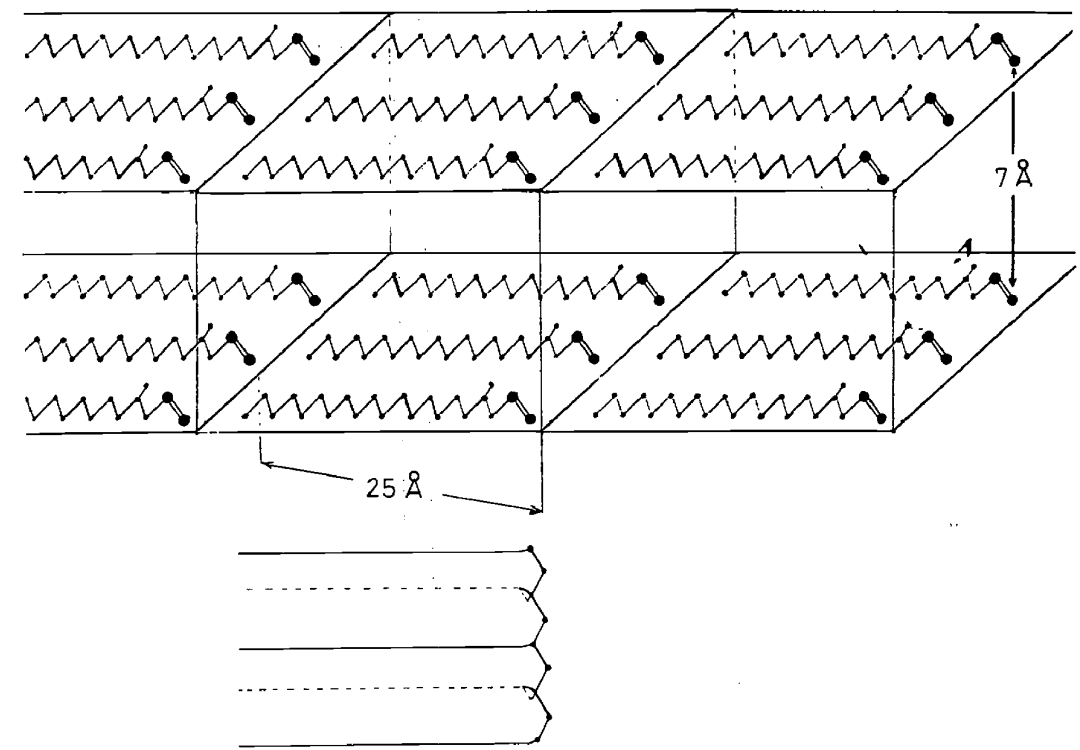

Figure 2. A schematic drawing of the crystal structure of vinyl stearate showing the initial separation of vinyl groups. The geometry of a syndiotactic polymer with side chains (lines) parallel to those in the monomer is shown in the lower portion of the drawing

that chain propagation will occur within, rather than between, these planes. In that case, the problem of determining the geometrical mechanism of chain propagation, effectively, is reduced to two dimensions. It is also reasonable to assume that polymerization can occur without appreciably disrupting the parallel arrangement of the large stearate side chains. The polymers generated 


\section{X-RAY STUDIES OF REACTIONS IN ORGANIC SOLIDS}

in this manner should have a syndiotactic geometry with side chains extending to the same side of the planes containing the polymer backbones.

Diffraction measurements indicated that a parallel and extended arrangement of the aliphatic side chains indeed was present in the polymer phase. However, a dilemma arose when it was discovered that the polymer was less syndiotactic than polymers obtained from the liquid state. Moreover, an isotactic geometry with side chains extending to both sides of the planes containing the polymer backbones was consistent with some observed crystallographic spacings. It is very difficult to conceive of any way in which the long stearate side chains can move through the very large distances required to produce a polymer of this geometry.

Similar conceptual difficulties in interpreting the molecular motions during transformation arise in our studies of peroxide rearrangements (vide infra), where it is clear that 'before and after photographs' of the terminal ordered phases are inadequate measures of the transformation. However, the molecular relationships are clarified considerably when the transformation occurs in discrete steps.

\section{Solid state rearrangements}

An elegant study by Dame Kathleen Lonsdale and her associates illustrates the manner in which the intermediate stages in a transformation can be
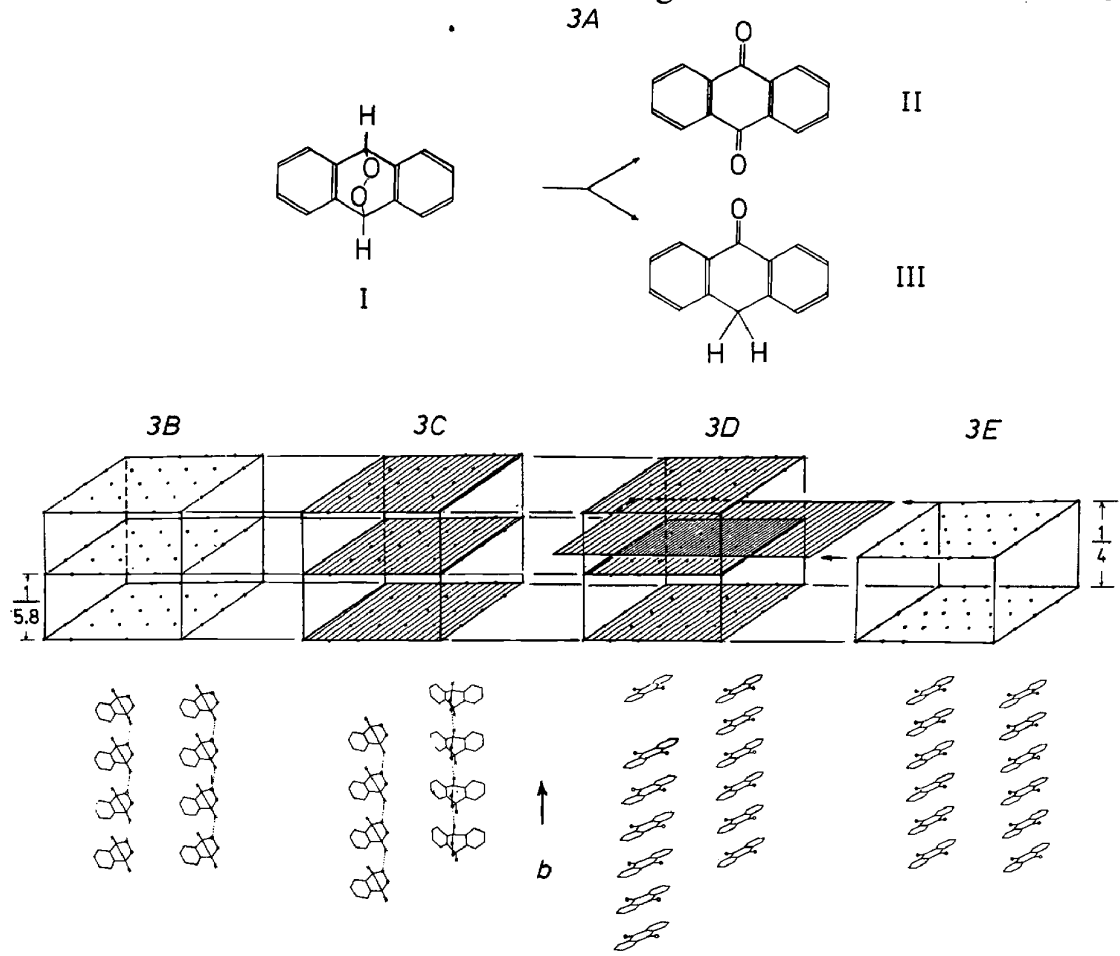

Figure 3. The successive stages in the decomposition of anthracene peroxide. A schematic representation of the behaviour of the molecular chains parallel to [010] is shown below the corresponding changes-B, $C, D, E$-in the reciprocal lattice 


\section{J. Z. GOUGOUTAS}

followed by $\mathrm{x}$-ray diffraction methods. Single crystals of anthracene peroxide, I, (Figure 3), upon moderate heating or continued exposure to $\mathrm{x}$-irradiation at room temperature, are transformed, without change in external shape, into mixed single crystals of anthraquinone, II and anthrone, III $^{8}$. Before reaction, the Bragg and Laue spots which correspond to structure I are well-shaped and apparently normal (Figure 3B). After a relatively brief period, there appear thin continuous sheets of diffraction passing through existing $k$-constant points of the peroxide reciprocal lattice (Figure $3 C$ ). These diffraction effects correspond to a mesomorphic phase consisting of chains of molecules parallel to the original $b$ lattice repeat. The molecular interval along each chain is constant and identical with the original peroxide spacing in this direction (5.8 $\AA$ ). However, there is no ordered relationship between individual chains; they may have rotated about the chain axis, moved sideways or shifted in the $b$ direction relative to one another. Upon further irradiation, new diffraction sheets appear in reciprocal space, parallel to and between the original sheets (Figure 3D). This second mesomorphic phase is similar to the first but the molecular interval along the chains has
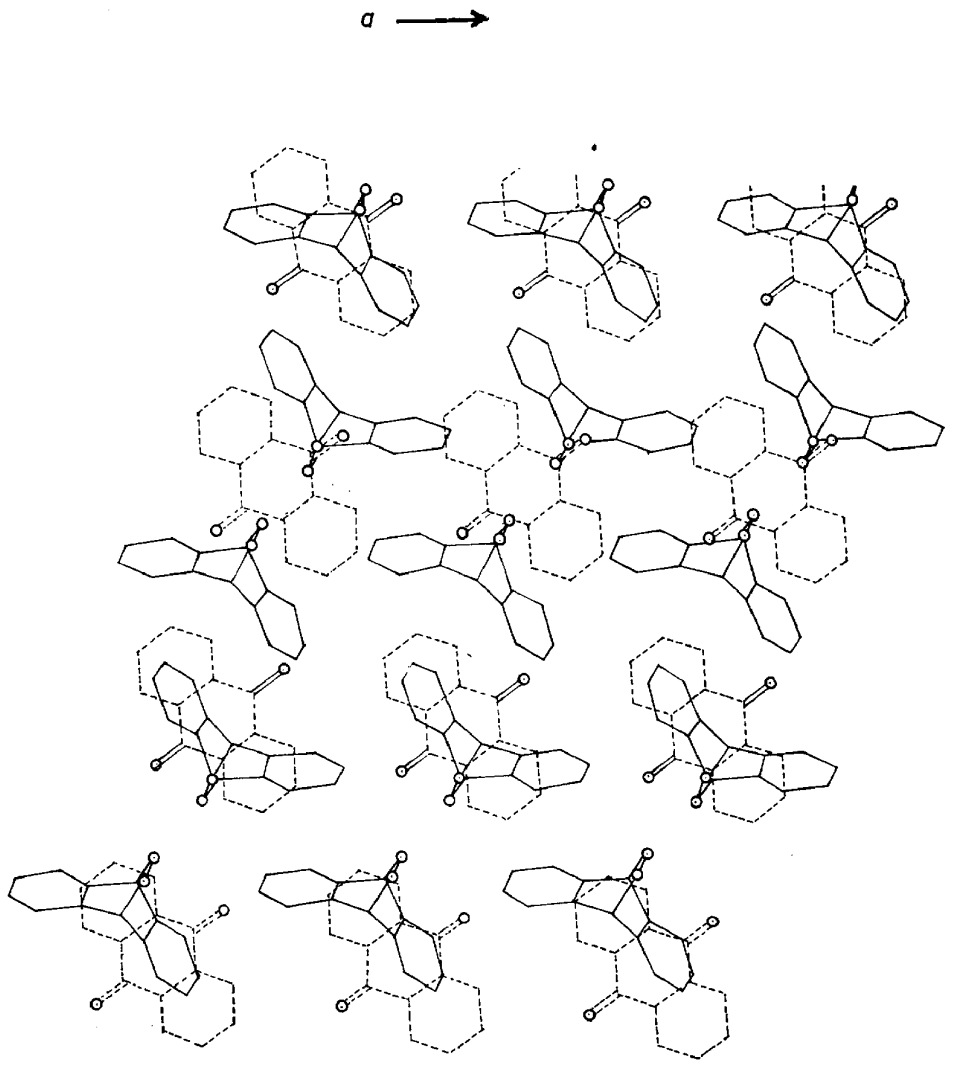

Figure 4. A possible superposition of the initial and final crystal structures in the decomposition of $I$. The invariant direction of the molecular chains, [010], is normal to the drawing.

(Courtesy of Prof. K. Lonsdale) 


\section{X-RAY STUDIES OF REACTIONS IN ORGANIC SOLIDS}

contracted to $4 \AA$. It was suggested that this change corresponds to chemical reaction. The chains of flat product molecules are formed within the mesomorphic peroxide chains and remain parallel to $b$. Finally, the continuous reciprocal lattice sheets which characterize the product chains are replaced by relatively sharp diffraction spots corresponding to the known II-III mixed crystal diffraction pattern (Figure $3 E$ ). In this stage, the chains of product molecules have crystallized to form the three-dimensionally ordered structure. The product phase is twinned in modes not previously observed in mixed crystals grown during solution crystallizations.

It is instructive to compare the initial and final structures. The possible superposition shown in Figure 4 was presented together with the comment that the reaction clearly requires large movements of the chains. In comparisons of this type, the experimentally observed angular orientation of the two structures must be retained. The superposition, however, is not uniquely defined and the two structures may be shifted in an infinite number of ways while preserving the angular orientation. The loss of crystallographic order normal to the chain axes suggests that no particular correspondence can be expected between reactant and product molecules in different chains. Nevertheless, the $a$ lattice repeats are nearly equal in length and essentially coincident. The molecular correspondence in other directions between the
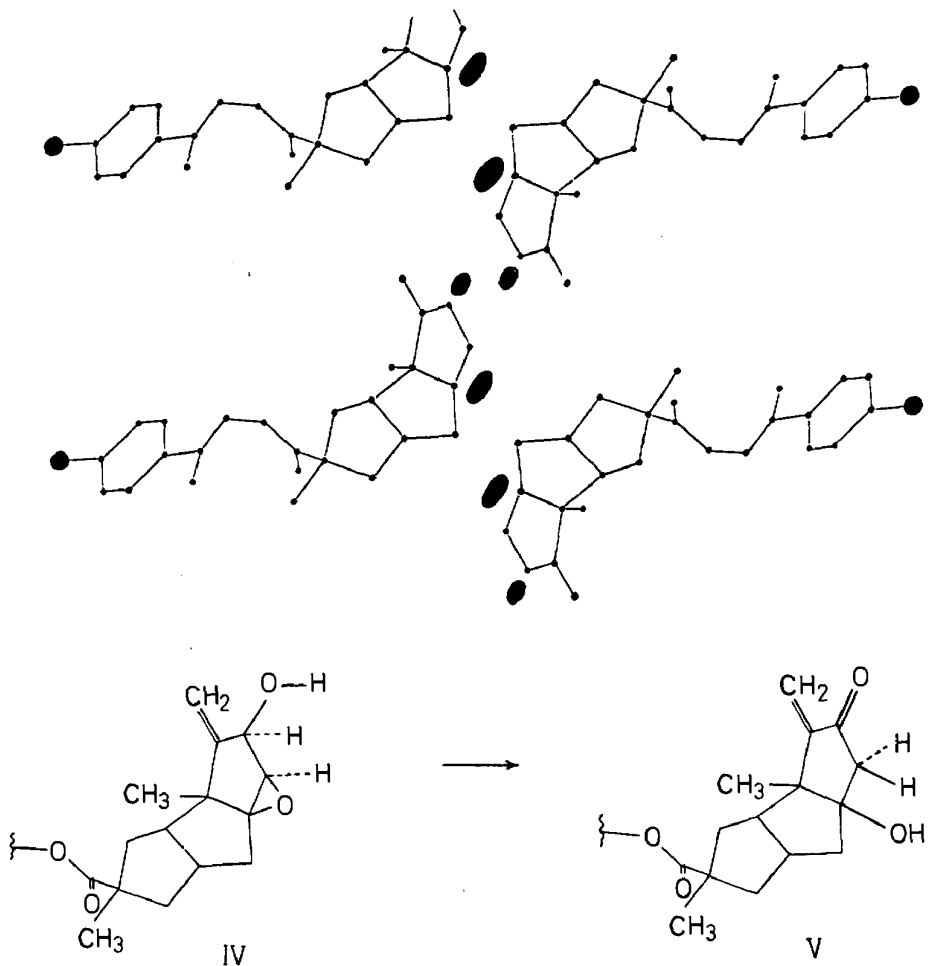

Figure 5. The molecular changes in the crystal structure of the $p$-bromophenacyl ester of hirsutic acid. 'Partial' oxygen atoms were found in the shaded regions 


\section{J. Z. GOUGOUTAS}

chains obviously is not simple. The product molecules along the chains may be identified with corresponding reactant molecules in the [010] direction. Even there, however, the chain contraction allows for an exact correspondence over only relatively short distances.

This method of assessing the significance of any apparent molecular correspondence between the initial and final structures, primarily, is one of elimination. It is thus possible to define the directions in which crystallographic order is not preserved. In the absence of experimentally observable disorder, however, the transformations appear three-dimensionally ordered throughout and the interesting possibility of direct transitions leading to an exact molecular correspondence should not be discounted.

A remarkably smooth isomerization was discovered during the crystal structure analysis of the $p$-bromophenacyl ester (IV) of hirsutic acid, a metabolite from stereum hirsutum ${ }^{9}$. This molecular rearrangement is a rare example of a topotactic reaction in which the product is not structurally incompatible with the reactant lattice and does not eventually appear in a separate phase. Indeed, a chemical reaction was not suspected until most of

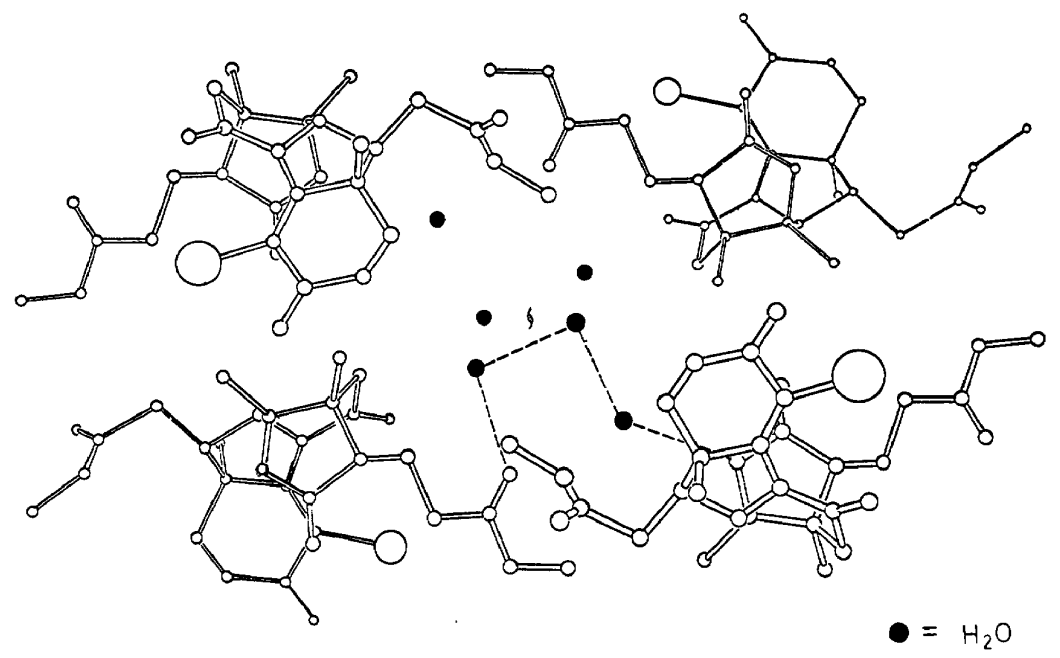

Figure 6. The crystal packing showing the unobstructed channels of water molecules; the molecular arrangement is repeated by simple translation along the normal to the drawing, [001]

the crystal structure had been revealed. The unsatisfactory progress of least squares refinements assuming structure IV was clarified when it was discovered that the final crystal structure was a solid solution of the isomers IV and $\mathrm{V}$ in the approximate ratio $2: 3$ respectively. Although the cell parameters changed by $\mathrm{ca} 1$ per cent during the transformation, presumably no significant changes in reflection intensities were observed. The solid state isomerization (Figure 5) requires the rupture and formation of three covalent bonds and the migration of at least two hydrogen atoms. The oxygen atoms involved in the reaction must move slightly from their initial positions. 
The initial homogeneity of crystalline IV and the unique radiation-induced isomerization were verified by independent chemical and spectroscopic methods. Walker later determined a lower limit of $G$ (isomerization) $\geqslant 57$ and suggested that this high value indicates either a chain reaction involving a chemically active propagating species or a highly efficient transfer process ${ }^{10}$.

It seems highly likely, in this case, that the large molecules remain at their original lattice positions throughout the transformation and a direct correspondence exists between the initial and final crystal structures. The terminal isomer ratio in the final pseudomorph supports this conclusion. Although hydrogen bonding is not possible in pure crystals of IV, it can occur between IV and V and between two molecules of V. The former hydrogen bonding accounts for the incomplete conversion of IV while the latter accounts for the predominance of $\mathrm{V}$. In view of this unexpected isomerization, the investigators recommend chromatographic analysis of samples after every crystal structure determination.

Miss Barbara Kaski in our laboratory observed a comparable transformation during the crystal structure analysis of a synthetic intermediate ${ }^{11}$. The initial crystal structure (Figure 6) contains water of crystallization which appears to be hydrogen bonded to the organic structure. The water molecules, originally contained within approximately cylindrical channels running throughout the crystal in the $c$ direction, may diffuse out of the crystal without appreciably disrupting the molecular packing of the large organic molecules. The small changes in cell parameters observed during the transformation suggest that a slight molecular reorganization occurs in order to fill the vacated channels.

\section{Reactions of 2-iodobenzoylperoxide derivatives $\dagger$}

A report that the isomerization of bis-(orthoiodobenzoyl) peroxide, VI, to the benziodoxole structure VII (Figure 7) occurs in the solid state ${ }^{12}$

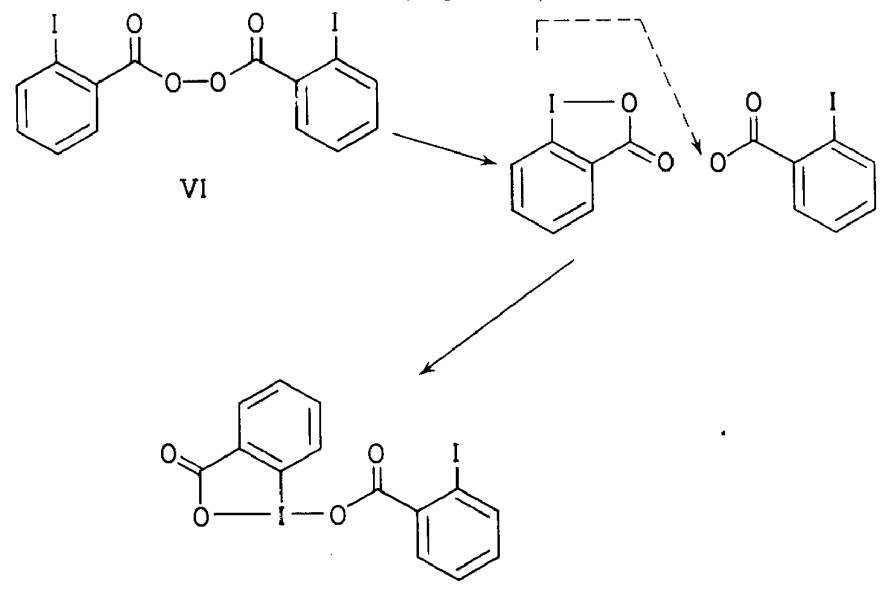

VII

Figure 7. The structural changes in the isomerization of bis-(orthoiodobenzoyl) peroxide

$\uparrow$ Financial support by the U.S. Air Force Office of Scientific Research (Grant No. AFOSR-69-1769) is gratefully acknowledged. 


\section{J. Z. GOUGOUTAS}

prompted us to investigate the reaction for possible topotaxy. Although the solid peroxide explodes when heated rapidly to $80^{\circ}$, rearrangement occurs smoothly during storage at room temperature for several weeks. The morphologically unchanged crystals are found to be twinned single crystals of the expected product, VII ${ }^{13}$. The same transformation also can be effected by heating overnight at $110^{\circ}$, after first maintaining the temperature at $50^{\circ}$ for several hours in order to prevent explosive decomposition. When the temperature is raised to $120^{\circ}$, the initially-formed crystal structure undergoes a polymorphic transformation to a polycrystalline pseudomorph.

VII, synthesized by an independent chemical route ${ }^{14}$, crystallizes from a variety of solvents in two polymorphic modifications ( $\alpha$ and $\beta$ ) (Table 1).

Table 1. Crystal data

\begin{tabular}{lrrrrrr}
\hline \multicolumn{1}{r}{ Structure } & $a(\AA)$ & $b(\AA)$ & $c(\AA)$ & $\beta(\mathrm{deg})$ & $\mathrm{z}$ & Space group \\
\hline VI & 13.05 & 4.21 & 15.47 & 121.1 & 2 & $P c$ \\
VII $\alpha$ & 4.21 & 30.86 & 22.52 & 93.3 & 8 & $C c$ \\
VII $\beta$ & 8.03 & 12.58 & 13.74 & 91.6 & 4 & $P 2_{1} / c$ \\
VIII & 4.32 & 15.08 & 11.29 & 91.5 & 4 & $P 2_{1} / c$ \\
IX & 12.89 & 4.10 & 14.05 & 96.7 & 4 & $P 2_{1} / c$ \\
X & 20.81 & 4.09 & 15.41 & 94.7 & 4 & $P 2_{1} / c$ \\
XI & 12.96 & 4.12 & 15.38 & 121.2 & 2 & $P c$ \\
XII & 13.00 & 4.08 & 15.38 & 120.9 & 2 & $P c$ \\
XIV & 22.28 & 4.06 & 15.38 & 94.5 & 4 & $P 2_{1} / c$ \\
XV & 4.28 & 24.34 & 6.89 & 91.3 & 2 & $P 2_{1}$ \\
XVI & 14.82 & 4.10 & 25.90 & 118.3 & 8 & $C 2 / c$ \\
\hline
\end{tabular}

The structural assignment as VII and the polymorphic relationship was verified through three-dimensional crystal structure analysis of both forms ${ }^{15}$. The dimorphs easily can be identified and separated: VII $\alpha$ is acicular with a

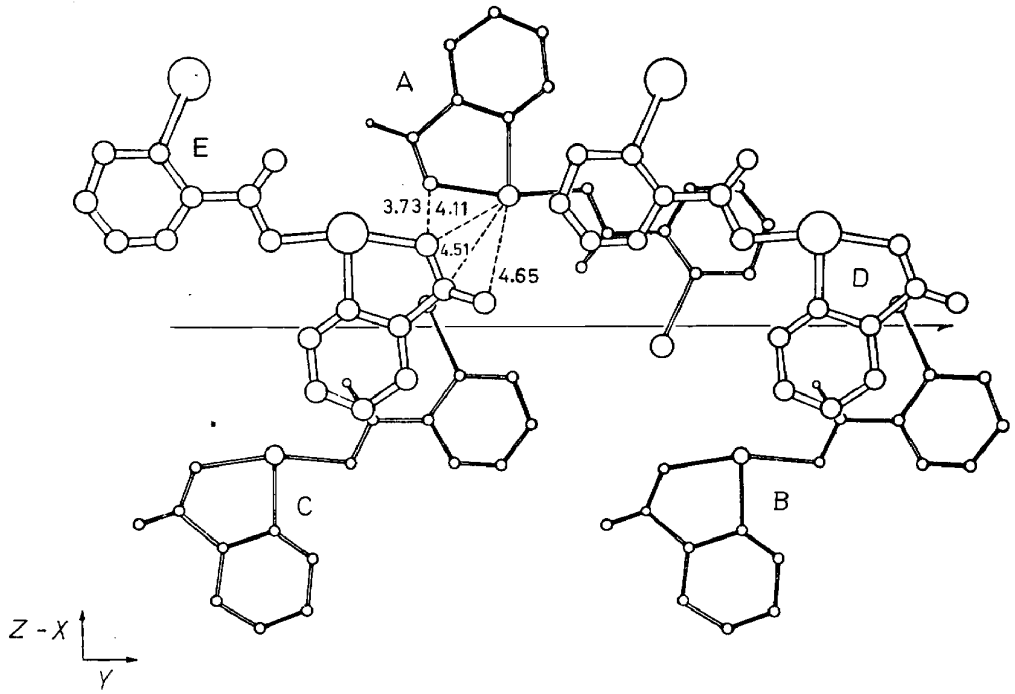

Figure 8. The molecular conformation and crystal packing of VII 
$4 \AA$ cell repeat parallel to the needle axis while crystals of VII $\beta$ are stout polyhedra. VII $\alpha$ (see Figure 12) is identical to the initial product phase from peroxide decomposition while VII $\beta$ (Figure 8 ) is the modification which results from the high temperature transition. The molecular conformation is markedly different in the two forms. The monovalent iodine atom and carboxyl oxygen atom of the iodobenzoate group adopt a cisoid relationship in VII $\beta$ while the presumably less stable transoid conformer ${ }^{16}$ occurs in VII $\alpha$.
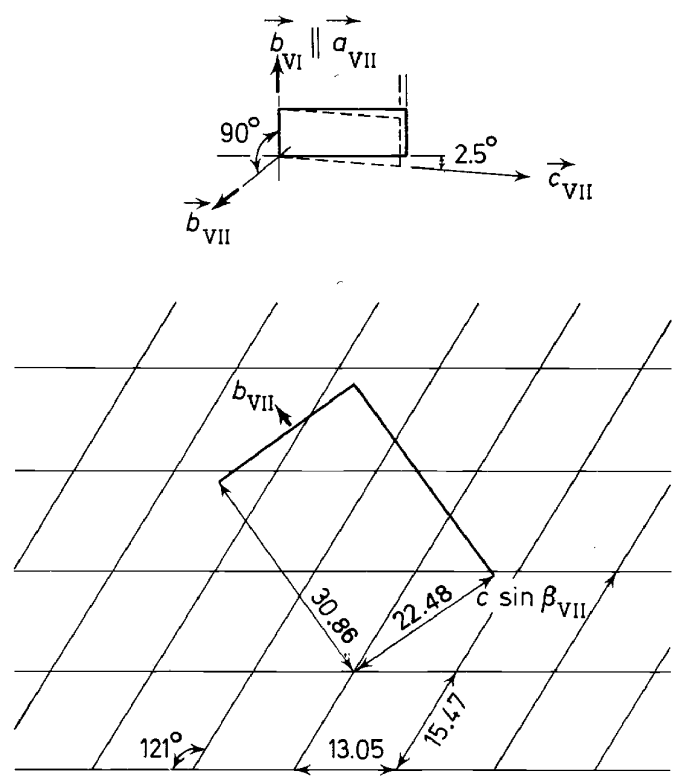

Figure 9. The geometrical phase relationship observed in the isomerization of VI. The lower drawing is a projection down [010] of VI and [100] of VII $\alpha$

The approximately flat peroxide molecules are stacked on top of one another in the initial layered crystal structure ${ }^{17}$ (see Figure 12 and Table 1). Although solid state isomerization is complete within a few days in the $\mathrm{x}$-ray beam at room temperature, the accelerating effect of $x$-irradiation does not alter the course of the reaction. The geometric relationship: $[010]_{\mathrm{V}_{I}} \|[100]_{\mathrm{v}_{\text {II }}}$ and $(001)_{\mathrm{VI}} \|(021)_{\mathrm{V} \text { II }}$ (Figure 9) was determined from x-ray photographs of intermediate states (Figures 10 and 11 ). The remarkably well-ordered product phase appears to be formed directly, without the intermediacy of mesomorphic phases. The relative orientation of the initial and final structures is shown in projection down the common short lattice repeat in Figure 12. Surprisingly large molecular displacements are indicated. All considerations of the geometrical mechanism of transformation must confront the unavoidable conclusion that half of the phenyl rings flip through $\mathrm{ca} 180^{\circ}$ about an axis parallel to the layers of peroxide molecules. It is difficult to imagine how 


\section{J. Z. GOUGOUTAS}

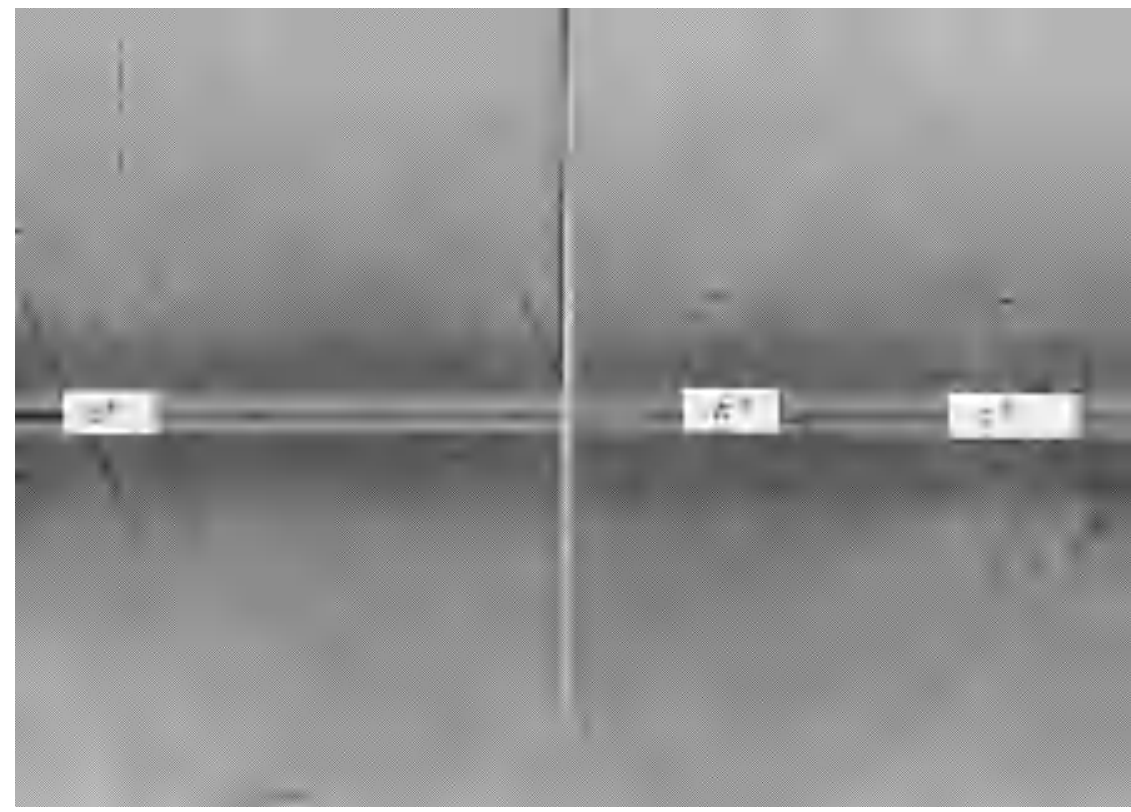

Figure 10. (Left) hOl Weissenberg photograph (Cu K $\alpha$ ) of a fresh crystal of VI after ca $20 \mathrm{hrs}$ exposure to $x$-rays at room temperature $\left( \pm a^{*}\right.$ are at the film extremes). Four successive 001 reflections of Vlla are clearly evident in the right half of the film. (Right) The same crystal after $\mathrm{ca} 100 \mathrm{hrs}$ in the $\mathrm{x}$-ray beam. (Crystal and camera settings have not been changed.) The discrete pattern is $0 k l$ of VIl $\alpha$. The extra circled reflections are due to newly-formed VIII

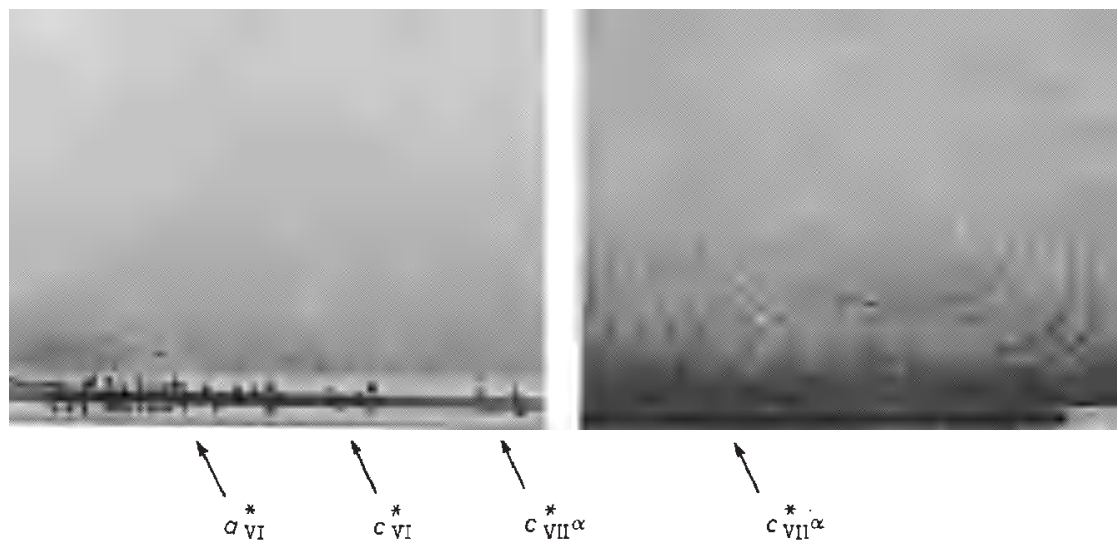

Figure 11. (Left) hll Weissenberg pholograph (Cu K $\alpha$ ) of VI (slightly mis-set) after ca 40 hrs total exposure to $\mathrm{x}$-rays at room temperature. The coplanar $1 k l$ net of VII $\alpha$ is clearly evident. (Right) The original $h I l$ net of VI has been replaced by the $I k l$ net of VII $\alpha$. Equal twinning across (001) of VIl $\alpha$, results in $\mathrm{mm}$ symmetry and an apparent doubling of $c^{*}$ 


\section{X-RAY STUDIES OF REACTIONS IN ORGANIC SOLIDS}

such rotations within the inadequate space between the layers can occur with retention of the interlayer spacingt.

Figure 13 shows the product phase partitioned into regions of 'flipped' and 'unflipped' iodobenzoate groups and it is clear that every molecule of
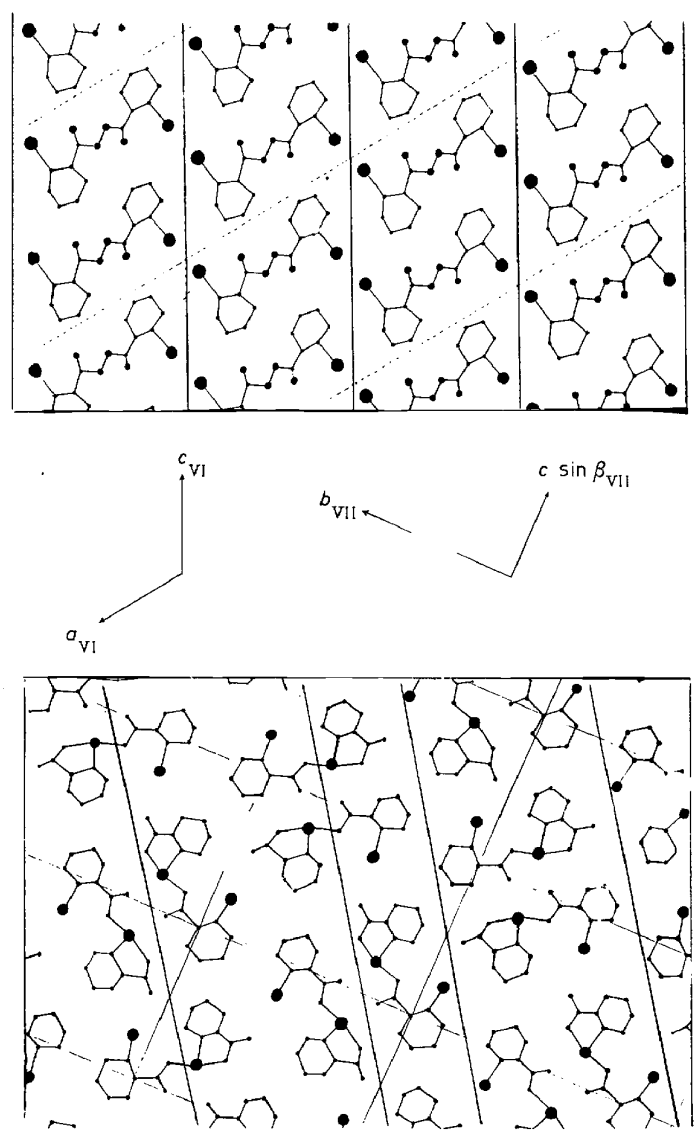

Figure 12. The molecular arrangements in the crystal structures of VI and VII $\alpha$. The structures, shown in projection down their common short lattice repeat, are oriented according to the observed geometrical relationship (see Figure 9)

product contains one of each. The dimensions, orientation and molecular distribution of this chequerboard pattern are reminiscent of the original peroxide structure and it is tempting to assume a simple correspondence. If a peroxide molecule originally lay wholly within one of these regions, the chemical mechanism is one of separation and intermolecular recombination. This being the case, either both or none of the phenyl rings in a given peroxide

† Similar rotations are necessary to interconvert the conformers of VII $\alpha$ and VII $\beta$. As mentioned above, the solid state transformation in that case completely disrupts the initial single crystal matrix. 


\section{J. Z. GOUGOUTAS}

molecule flip during the transformation. Alternatively, if the boundaries of the chequerboard pattern divide rather than contain original peroxide molecules, a mixed mechanism is operative. Half of the peroxide molecules rearrange intramolecularly while the others separate and recombine intermolecularly. Several chemical and crystallographic rationales for these and other mechanisms have been considered but none seem entirely satisfactory.

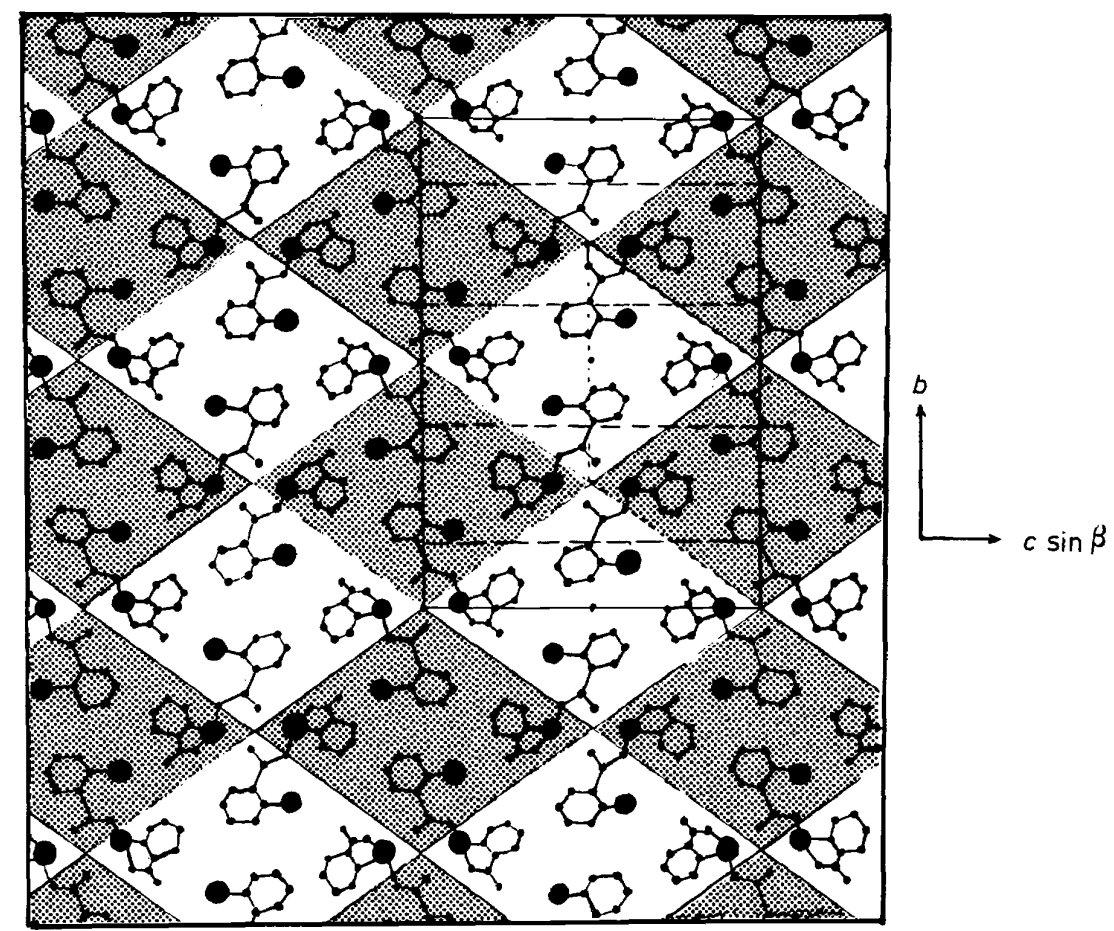

Figure 13. The crystal structure of VII $\alpha$ partitioned into regions of 'flipped' and 'unflipped' iodobenzoate groups

Further study of the initial pseudomorph revealed a second, consecutive topotactic reaction. The initial diffraction spectrum of VII $\alpha$ is slowly replaced by the single crystal spectrum of $o$-iodobenzoic acid ${ }^{15}$, VIII (Figure 14). The increased background level and rather streaked reflections suggest a somewhat fibrous crystal with considerable amorphous structure. Again, the external shape of the crystal is unchanged. The crystal structure of VIII also contains a $4 \AA$ lattice repeat which, in the solid state reaction, is generated parallel to the short repeats of VI and VII $\alpha$. The mutual orientations of VI, VII $\alpha$, and VIII are shown in Figure 15. No obvious correlation exists between VII $\alpha$ and VIII even though it is not necessary to invoke 'flipping' during the second transformation. Striking similarities are evident between the initial peroxide structure and the twice-removed acid structure. It appears that half of the original halobenzoate groups have remained virtually stationary while 


\section{X-RAY STUDIES OF REACTIONS IN ORGANIC SOLIDS}
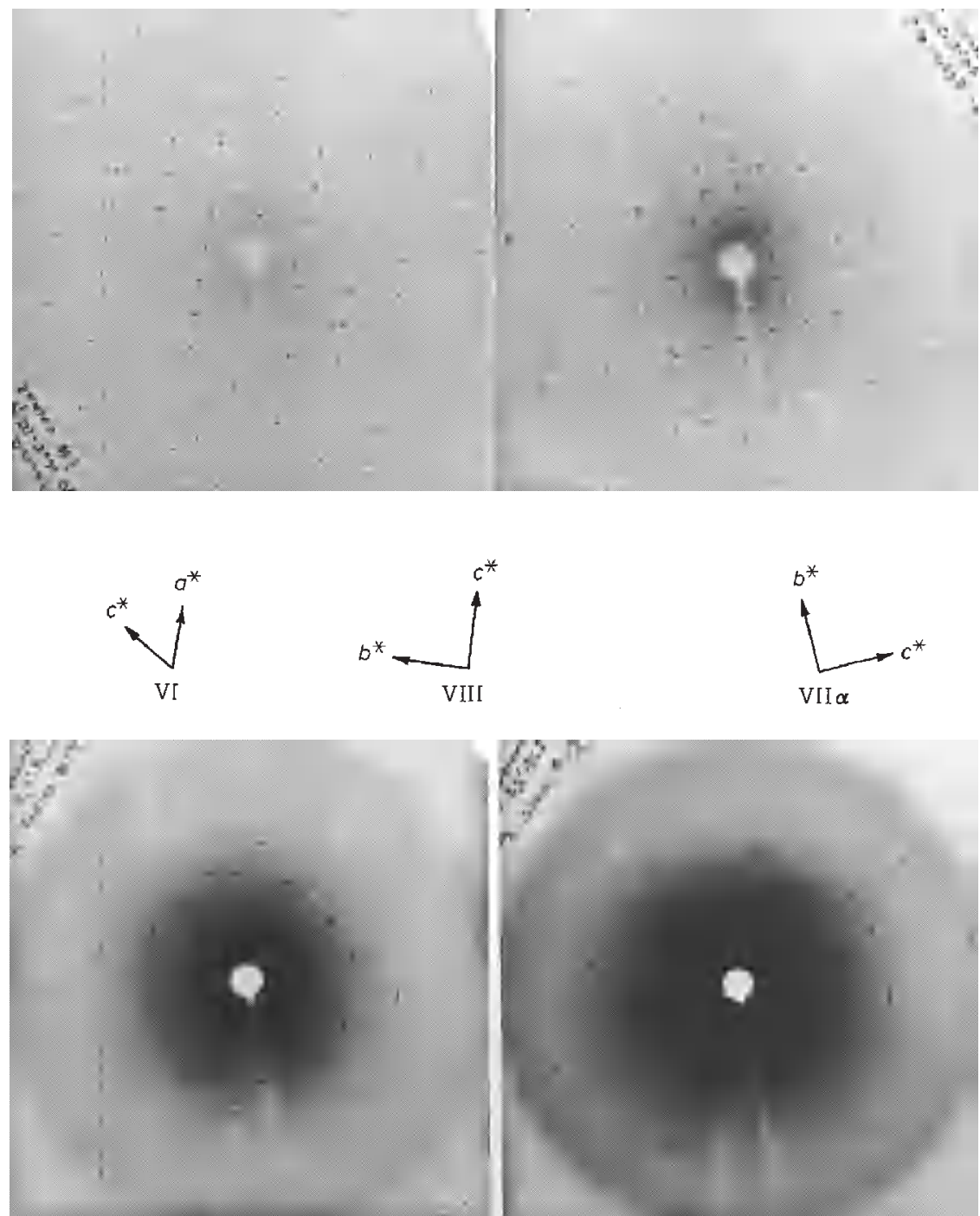

Figure 14. A series of precession photographs ( $\mathrm{Cu} \mathrm{K} \alpha$ ) of successive stages in the transformations $\mathrm{VI} \rightarrow \mathrm{VII} \alpha \rightarrow$ VIII of one crystal. (Identical experimental settings.) (Top Left) hol of freshly crystallized VI; (Top Right) $0 k l$ of VII $\alpha$. A few discrete reflections from VI are still visible. (Bottom Right) (ca three weeks after stage two.) Reflections of the $0 \mathrm{kl}$ zones of both VII $\alpha$ and VIII are present.(Bottom Left) (ca three weeks after stage three) $0 k l$ reflections of VIII. A few intense reflections of VII $\alpha$ have not yet disappeared 


\section{J. Z. GOUGOUTAS}

the others have separated and 'flipped'. This relationship probably is fortuitous since the acid appears well after the disappearance of a discrete peroxide phase. The direct solid state conversion VI $\rightarrow$ VIII has never been observed.

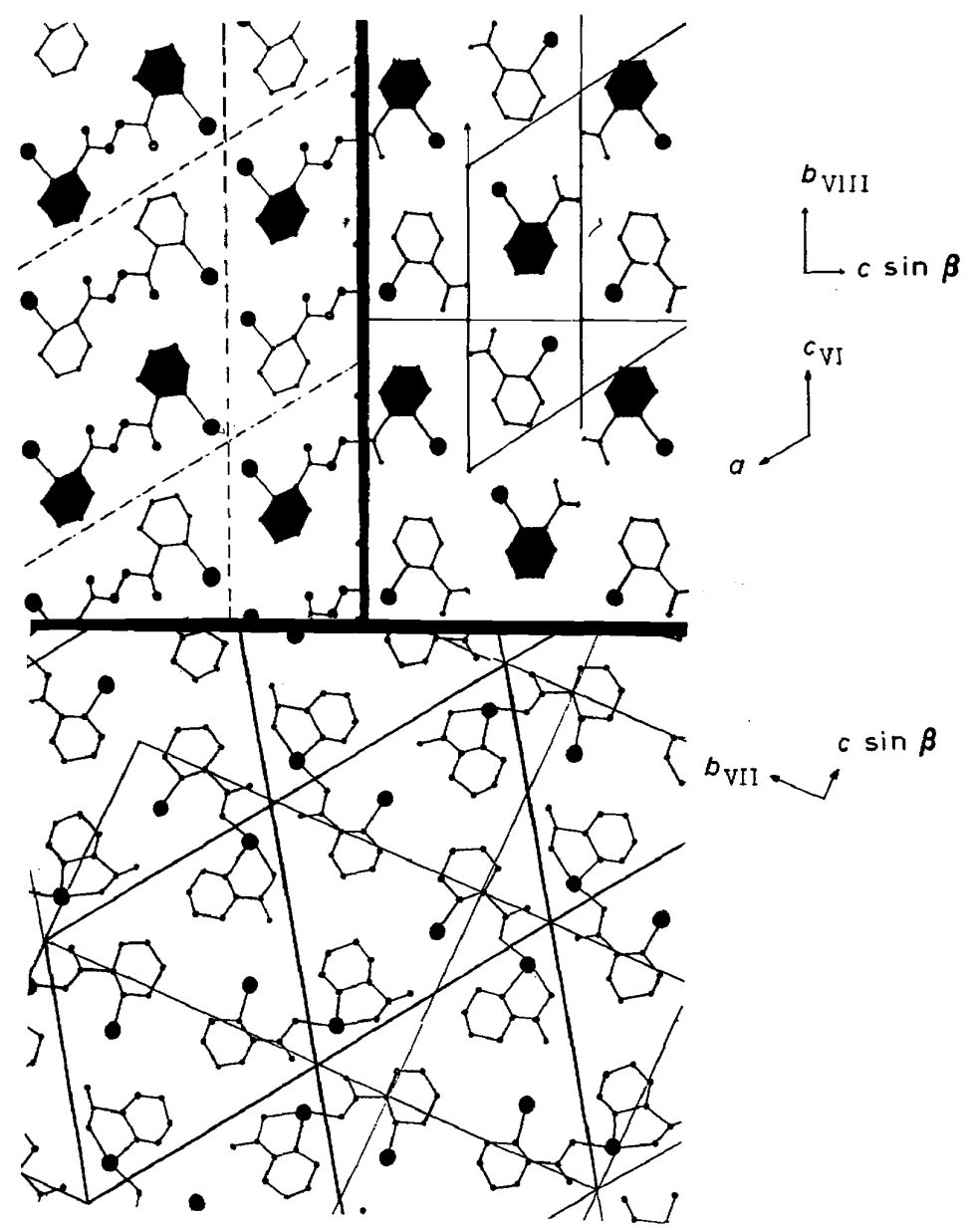

Figure 15. The experimentally observed mutual orientations of VI, VII $\alpha$ and VIII are shown in projection down their common short lattice repeat. Heavy lines separate the structures. Lighter lines within each structure are traces of several different planes which are qualitatively useful in comparing the molecular distributions

The initially-formed opaque pseudomorph contains numerous defects and readily admits atmospheric moisture for the subsequent hydrolysis reaction. By contrast, crystals of VII $\alpha$ from solution crystallizations do not undergo the transformation VII $\alpha \rightarrow$ VIII at any appreciable rate. Hydrolysis of VII in various solvents gives an equal mixture of VIII and $o$-iodosobenzoic acid, IX. 


\section{X-RAY STUDIES OF REACTIONS IN ORGANIC SOLIDS}

Although an ordered structure of IX often is observed in topotactic reactions of other related peroxides (vide infra), in this case it presumably occupies the amorphous regions of the pseudomorph. Solid state recrystallization after hydrolysis necessitates major molecular reorganization which nullifies any molecular correlations involving VIII. After several months, well-formed transparent needles of VIII grow out of the pseudomorph strictly parallel to [010] of the original peroxide crystal.

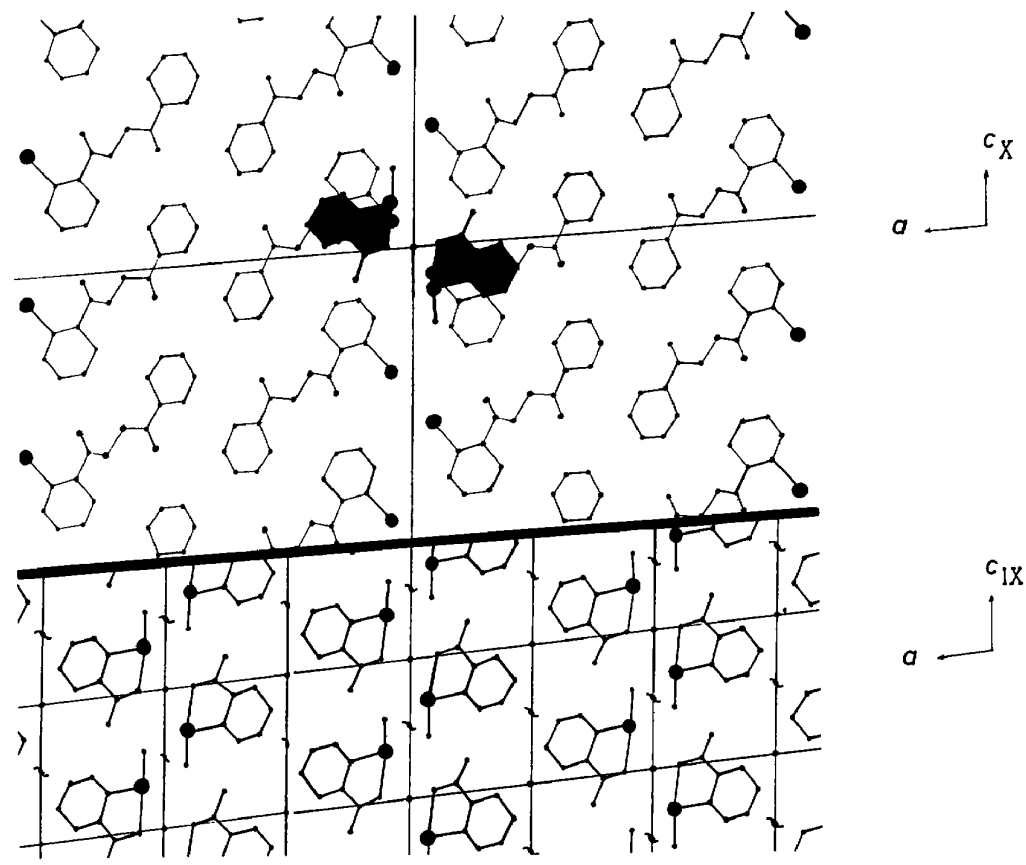

Figure 16. The experimentally observed orientation of initial and final crystal structures in the transformation $X \rightarrow I X$. [010] of both structures is normal to the drawing. The solid structures in $\mathrm{X}$ are oriented molecules of IX

The solid state chemistry of several other related peroxides was explored in order to examine the generality of the above transformations: X, 2iodobenzoyl peroxide; XI, 2-iodo-2'-bromobenzoyl peroxide; XII, 2-iodo-2'chlorobenzoyl peroxide; XIII, 2-iodo-2'-fluorobenzoyl peroxide; XIV, 2-iodo-3'-chlorobenzoyl peroxide; XV, 2-iodo-4'-nitrobenzoyl peroxide. These peroxides have layered crystal structures with a short cell dimension of $4 \AA$ (Table 1). With the interesting exception of XV, the molecular packing within the layers is similar to that in VI. Although all of the peroxides decompose in the solid state, no topotaxy has been observed in the case of XV.

The others undergo several competing and consecutive topotactic transformations which cannot be fully described in this presentation. Some typical processes are exemplified by the extraordinary behaviour of XI, which is isostructural with VI. The corresponding benziodoxole isomer is dimorphic 


\section{J. Z. GOUGOUTAS}

but neither form is isostructural with either VII $\alpha$ or VII $\beta$. During reaction, an interphase perpendicular to the crystal needle axis $(b)$ often can be observed to progress along the length of the crystal. X-ray studies of crystals cleaved at the boundary demonstrate that one part is product and the other, unchanged peroxide. Either one, or the other, and often both crystal structures of the isomeric product are formed directly as single crystal phases. Once again, half of the phenyl rings of the peroxide phase 'flip' during the transformation $\dagger$ and no clear molecular correspondence is indicated between the initial and final structuresł. This product is hydrolyzed by atmospheric moisture to $o$-iodosobenzoic acid, IX and $o$-bromobenzoic acid, XVI. Unlike the hydrolysis of VII $\alpha$, however, both of the acidic products crystallize in the pseudomorph as discrete oriented phases of known crystal structure ${ }^{18,19}$.

The direct topotactic formation of IX from a peroxide crystal structure has been observed in decompositions of X and XIV. In the case of the former peroxide, it is also the only crystalline product. Benzoic acid, the major product from the other half of the peroxide molecule, is contained in amorphous regions of the pseudomorph. The extensive molecular reorganization which must ensue can be inferred from the experimentally observed phase relationship (Figure 16). If hydrolysis of $\mathrm{X}$ occurs through the addition of water to the more electrophilic and less hindered carboxyl group, benzoic acid and 2-iodoperbenzoic acid, XVII, should be formed. The latter appears to be unstable with respect to its cyclic isomer, IX $\%$. In this connection, the direct hydrolysis of XI (and XII) is highly interesting.

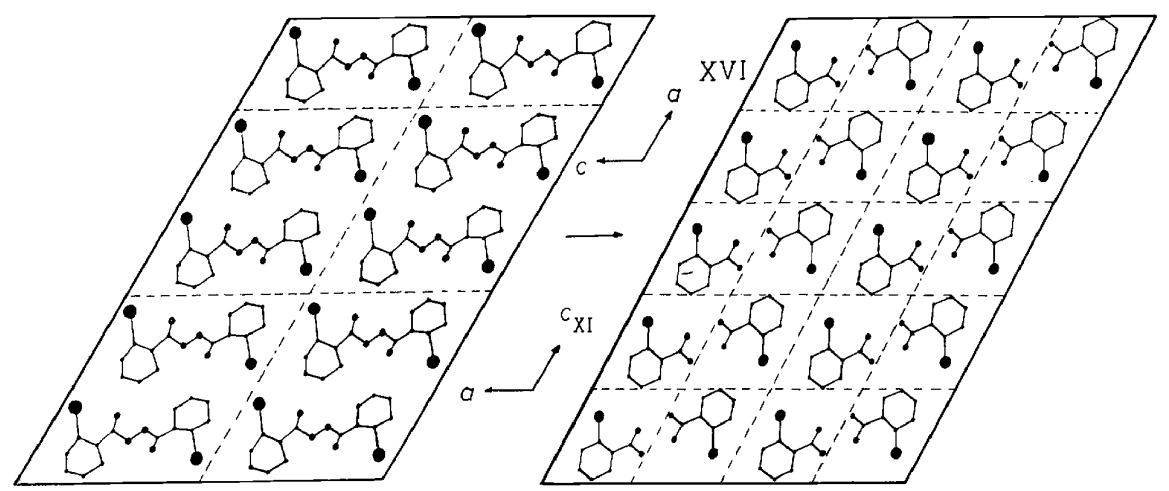

Figure 17. The experimentally observed orientation of the initial and final crystal structures in the transformation XI $\rightarrow$ XVI. [010] of both structures is normal to the drawing

The specific behaviour of XI is variable and depends on the ambient temperature and humidity as well as the history of the particular crystal specimen. Direct hydrolysis competes with molecular isomerization and

$\dagger$ The molecular conformation is transoid (vide supra) in both dimorphs. In this case, 'flipping' is necessary for the formation of both product crystal structures.

$\ddagger$ Indeed, the single crystal spectrum of VII $\alpha$ has been observed in some decomposing crystals of XII! Peroxides XI and XII are isostructural, as are their corresponding benziodoxole isomers.

If Attempted syntheses of XVII by conventional methods ${ }^{20,21}$ yield IX. 
often the formation of the oriented structure of XVI precedes the appearance of single crystal reflections from the benziodoxole phase. The striking geometrical correspondence in the direct topotactic transformation XI $\rightarrow$ XVI (Figure 17) is not relevant to the chemical reaction since only the bromobenzoate groups of the peroxide appear in the acid phaset. Frequently, a second, relatively unstable single crystal phase appears in conjunction with XVI. The diffraction spectrum of the former, as yet unidentified phase is similar to that of IX, and VII $\alpha$ and may represent either the elusive crystal structure of XVII or a third, metastable modification of the benziodoxole product. Both alternatives are particularly noteworthy since the other topotactic transformations simply duplicate crystal structures which are obtained from conventional crystallizations. The significant possibility that topotaxy is instrumental in the formation of a unique crystal structure cannot be excluded $\ddagger$.

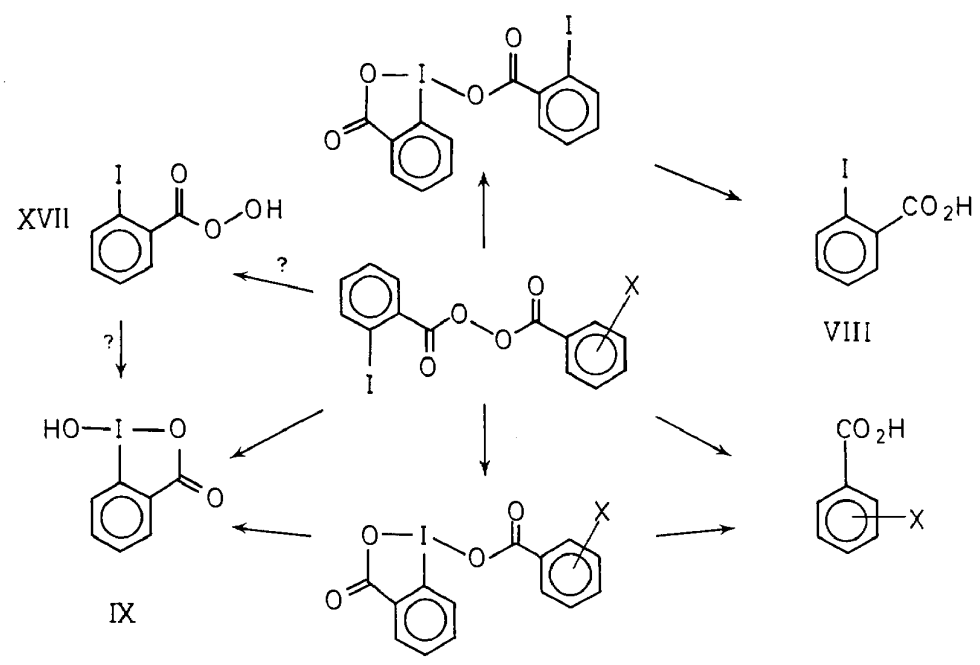

Figure 18. Solid state chemical reactions of 2-iodobenzoyl peroxide derivatives. (For a review of polyvalent iodine compounds, see reference 24)

The vicissitudes of these transformations (Figure 18) can be appreciated in the x-ray photograph of a decomposing crystal of XI (Figure 19). At least seven discrete single crystal phases have been identified. The elucidation of the individual crystal structures and the complicated topotactic reactions of these peroxides reflects the unusual skill of my collaborators in this workJ. C. Clardy, A. M. Glazer, L. Lessinger, S. Singh and R. Weiss.

$\uparrow$ 2-Iodobenzoic acid has an entirely different crystal structure and a solid solution seems unlikely $^{22}$.

$\ddagger$ Uncommon crystal structures frequently are formed in reactions which yield $\beta$-truxinic acids $^{23}$. It is not clear, however, whether these phases are unique to the solid state process or simply solvent free modifications which also could be obtained by other methods. 


\section{J. Z. GOUGOUTAS}

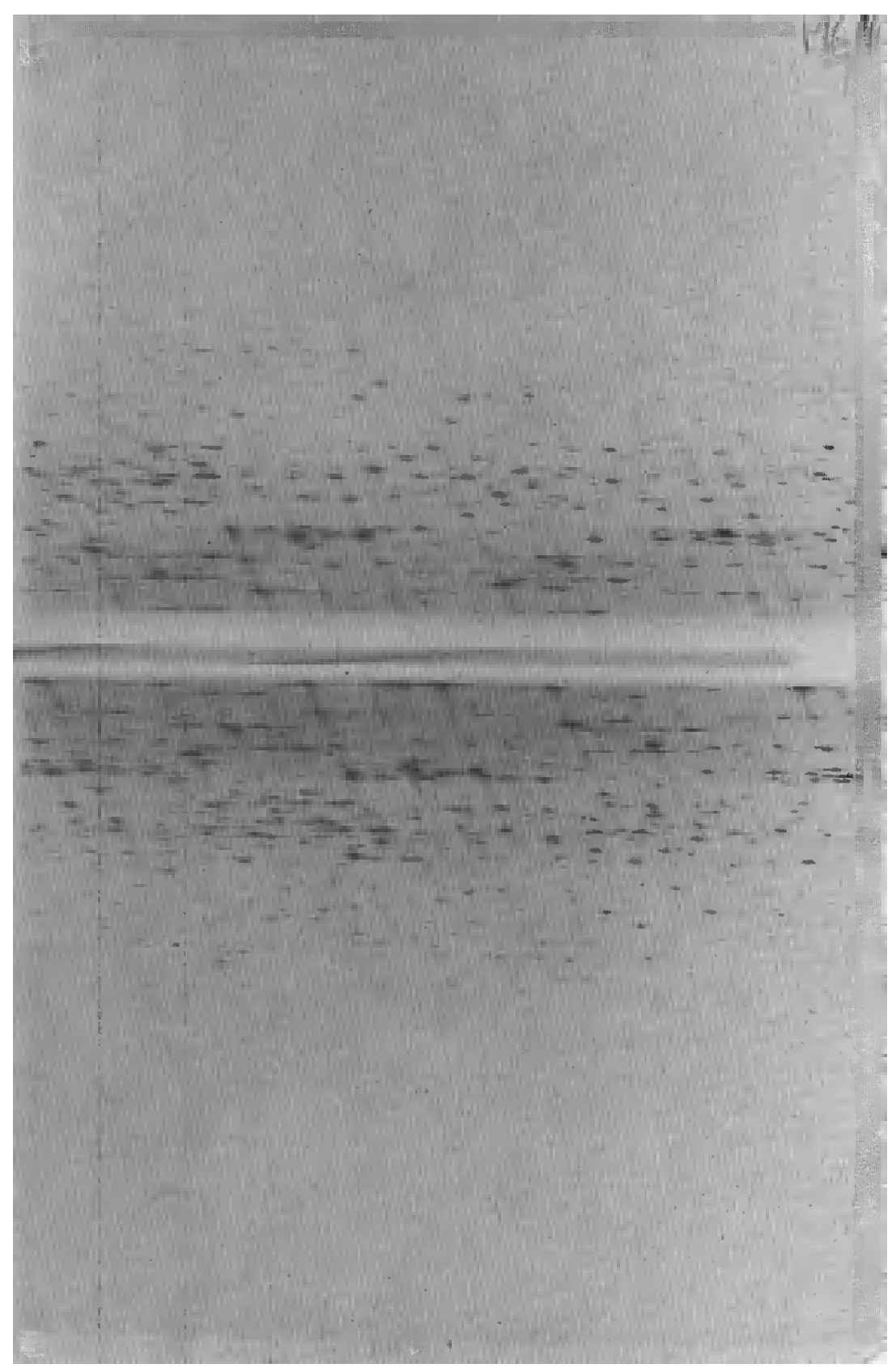

Figure 19. Weissenberg photograph $(\mathrm{Cu} \mathrm{K} \alpha)$ of a decomposing single crystal of XI mounted along [010]. Sharp, discrete hOl reflections from XI are easily identified in the centre of the film at low angles of diffraction. The diffraction spectrum initially resembled that in Figure 10 (left) 


\section{CONCLUSION}

Finally, I must apologize for oversimplifying both the positive and negative results of the above investigations. A discussion of the quantitative reticular correspondence was postponed in order to concentrate on the molecular aspects of the transformations. The interdependence of the two, however, appears to be quite subtle. The topotaxy and epitaxy between the various phases presumably defines a unique assembly of crystal structures and interphases. Crystallographic ordering is obvious but minimal motion is by no means a critical requirement. This seems less to be the case in the equally novel twinned product structures where selective restrictions of the different types of molecular reorientation, necessary to form the individual members, are indicated ${ }^{25}$. In virtually all cases, however, the short-range molecular arrangement simply duplicates that formed in an unrestricted milieu.

The study of the molecular motions during transformation is crucial to an understanding of these interrelationships. Additional evidence is necessary when only the initial and final structures are available for study by $x$-ray methods. Where possible, identical or different types of topotaxy and, in some cases its conspicuous absence, should be considered for the same chemical reaction in polymorphic forms of the molecular reactant. The behaviour of crystallographically isostructural reactants of inherently different chemical reactivity is also relevant to this problem.

\section{REFERENCES}

1 M. D. Cohen, G. M. J. Schmidt et al. J. Chem. Soc. 1966 (1964).

2 L. S. Dent Glasser. F. P. Glasser and H. F. W. Taylor. Quart. Rev. (London) 16, 343 (1962).

${ }^{3}$ H. Morawetz. Pure and Appl. Chem. 12, 201 (1966).

4 See, for example: S. Okamura, K. Hayashi and Y. Kitanishi. J. Polymer Sci. 58, 925 (1962); G. Carazzolo, S. Leghissa and M. Mammi. Makromol. Chem. 60, 171 (1963); N. Morosoff, D. Lim and H. Morawetz. J. Am. Chem. Soc. 86, 3167 (1964).

5 J. B. Lando and V. Stannett. J. Polymer Sci. Part A 3, 2369 (1965).

${ }^{6}$ G. Carazzolo and G. Valle. Makromol. Chem. 90, 66 (1966).

7 N. Morosoff, B. Post and H. Morawetz. J. Am. Chem. Soc. 87, 3035 (1965).

8 K. Lonsdale, E. Nave and J. F. Stephens. Phil. Trans. Roy. Soc. London A 261, 1 (1966).

${ }^{9}$ F. W. Comer and J. Trotter. J. Chem. Soc. B, 13 (1966).

10 D. C. Walker. Chem. Commun. 1050 (1967).

11 B. Kaski. Ph.D. Thesis. Harvard University (1970).

12 J. E. Leffler, R. D. Faulkner and C. C. Petropoulos. J. Am. Chem. Soc. 80, 5435 (1958).

13 J. Z. Gougoutas, J. C. Clardy, A. M. Glazer, L. Lessinger and S. Singh. Acta Cryst. A 25, S 232 (1969).

14 W. Honsberg and J. E. Leffler. J. Org. Chem. 26, 733 (1961).

15 J. C. Clardy. Ph.D. Thesis. Harvard University (1969).

16 G. Eglinton, G. Ferguson, K. M. S. Islam and J. S. Glasby. J. Chem. Soc. B 1141 (1967).

17 J. Z. Gougoutas and J. C. Clardy. Acta Cryst. B26. 1999 (1970).

18 E. Shefter and W. Wolf. J. Pharm. Sci. 54, 104 (1965).

19 G. Ferguson and G. A. Sim. Acta Cryst. 15, 346 (1962).

20 L. S. Silbert, E. Siegel and D. Swern. J. Org. Chem. 27, $1336(1962)$.

21 J. R. Moyer and N. C. Manley. J. Org. Chem. 29, 2099 (1964).

22 A. I. Kitaigorodskii, Kristallografiya 2, 456 (1957); also Dokl. Akad. Nauk. SSSR 113, 604 (1957).

23 G. M. J. Schmidt. J. Chem. Soc. 2014 (1966).

24 D. F. Banks. Chem. Rev. 66, 243 (1966).

${ }^{25}$ K. Lonsdale. Acta Cryst. 21, 5 (1966). 